\title{
CONDENSED-MATTER SPECTROSCOPY
}

\section{Charge-Transfer Transitions and Optical Spectra of Chromites}

\author{
A. V. Zenkov \\ Ural Federal University, Ekaterinburg, 620002 Russia \\ e-mail:andreas@k66.ru \\ Received August 2, 2012
}

\begin{abstract}
Specific features of charge-transfer states and charge-transfer transitions of the $\mathrm{O}_{2 p} \rightarrow \mathrm{Cr}_{3 d}$ type in octahedral complexes $\left(\mathrm{CrO}_{6}\right)^{9-}$ have been considered in the cluster approximation. Reduced matrix elements of the electric-dipole moment operator on many-electron wave functions, which are the initial and final states for charge-transfer transitions, are calculated. The results are parameterized, and the relative intensities of different allowed charge-transfer transitions in the absence of mixing of different charge-transfer configurations with identical symmetry are calculated. This mixing is taken into account within the Tanabe-Sugano theory, and the true energies and intensities of many-electron charge-transfer transitions are obtained. The Coulomb interaction between $2 p$ electrons of $\mathrm{O}^{2-}$ ligands and $3 d$ electrons of the central $\mathrm{Cr}^{3+}$ ion in $\left(\mathrm{CrO}_{6}\right)^{9-}$ cluster is considered. The influence of this interaction on the optical spectra is found to be insignificant. Simulation of the optical spectra of chromium oxides has shown the presence of a band of complex charge-transfer transitions composed of 33 lines with a total width of about $8 \mathrm{eV}$. The model spectrum is in adequate agreement with the experimental data, which indicates limited applicability of the widespread view that charge-transfer transition spectra have a simple structure.
\end{abstract}

DOI: $10.1134 / \mathrm{S} 0030400 \mathrm{X} 13030302$

\section{INTRODUCTION}

The discovery of high-temperature superconductivity in $R \mathrm{OFeAs}$ systems ( $R$ is a rare earth metal), as in cuprates two decades ago, and finding of giant magnetoresistance in systems based on $\mathrm{Mn}^{3+}$ ions, has increased interest in $3 d$ oxides. The nature of their electronic structure and energy spectrum remains an important problem of the physics of these strongly correlated systems. Optical and magneto-optical methods are very appropriate for their study; however, the nature of low-energy electron-hole excitations in $3 d$ oxides is still debated.

The following concept was proposed for ferrite garnets [1] (and extended later to the wider class of ironcontaining oxides [2]): their optical and magnetooptical properties in the near-UV range and the fundamental optical absorption edge are determined by charge-transfer transitions of the $\mathrm{O}^{2-} \rightarrow 3 d$ ion type. This idea was developed further in [3, 4]; now it is a well-accepted concept of electron spectroscopy [5].

However, there is no exhaustive theory of chargetransfer transitions in $3 d$ oxides. In most studies, these transitions are considered practically from the same viewpoint as in the first investigations. Specific features of optical spectra of $3 d$ oxides are generally related to charge-transfer transitions of the $2 p \longrightarrow 3 d$ type (note that indication of electron shell does not set unambiguously the wave function!). In the best case, the $t_{2 g}, e_{g}$ classification of $d$ electrons in (cubic) crystal field is taken into account and $2 p \rightarrow 3 d t_{2 g}$ and $2 p \rightarrow 3 d e_{g}$ transitions are considered [6]. This is the single-electron approximation for charge-transfer states and transitions, based on which one cannot even qualitatively explain the optics of $3 d$ oxides.

The above-stated problem cannot be solved using single-electron band models (including the LDA $+U$ model), because they neglect important effects of intra-atomic correlations, which are responsible for the fine structure of the terms of ground and excited charge-transfer states.

We will consider charge-transfer states and transitions in chromites in the relationship with their optical spectra within the cluster model of one-center chargetransfer transitions. This model has showed its advantage in consideration of the optics and magnetooptics of orthoferrites, ferrite garnets, and other $3 d$ oxides [7-10]. It is characterized by physical clarity and possibility of taking into account electron-electron correlations and crystal-field effects.

\section{CHARGE-TRANSFER STATES AND TRANSITIONS IN CHROMITES}

Weakly deformed octahedral $\left(\mathrm{CrO}_{6}\right)^{9-}$ complexes are the main optical and magneto-optical centers in perovskite-type $R \mathrm{CrO}_{3}$ oxide compounds ( $R$ is a rare earth or yttrium cation). 
The ground electronic configuration of $\left(\mathrm{CrO}_{6}\right)^{9-}$ complex includes filled molecular orbitals (MOs) $\mathrm{O}_{1 s, \ldots, 2 p}$ of basically anionic nature, filled (basically cationic) MOs, and MOs of $3 d$ type. The ground state of the complex is the orbital singlet $\left|\kappa_{0} \gamma_{2 p}^{6} t_{2 g}^{3}{ }^{4} A_{2 g}\right\rangle$, where $\kappa_{0}$ denotes intrinsic MOs, which do not change at a charge-transfer transition; these MOs are omitted below.

An excited configuration of charge-transfer complex arises when an electron passes from an "anionic" MO $\gamma_{2 p}\left(t_{2 u}(\pi), t_{1 u}(\pi), t_{1 u}(\sigma)\right)$ to a $3 d$-type MO $\left(t_{2 g}\right.$ or $e_{g}$ ) of the central ion of $\mathrm{Cr}^{3+}$ complex. The chargetransfer configuration can be presented as $\tilde{\gamma}_{2 p}^{1} 3 d^{4}$, where $\tilde{\gamma}_{2 p}^{1}$ denotes a hole in the anionic framework. The transition between the ground and excited configurations is a charge-transfer $\gamma_{2 p} \rightarrow 3 d\left(t_{2 g}, e_{g}\right)$ transition.

The charge-transfer configuration contains two unfilled shells: a "ligand" $\gamma_{2 p}$ shell and a $3 d$ shell. With the interaction between a $\tilde{\gamma}_{2 p}^{1}$ hole with $3 d$ electrons neglected, the many-electron states of the chargetransfer configuration are recorded as $\left|\tilde{\gamma}_{2 p}^{12}{ }^{2} \Gamma_{u} ; 3 d^{42 s^{\prime}+1} \Gamma_{g}^{\prime}\right\rangle$. The Coulomb interaction of $2 p-3 d$ type splits these states into $\left|\tilde{\gamma}_{2 p}^{1}{ }^{2} \boldsymbol{\Gamma}_{u} ; 3 d^{42 s^{\prime}+1} \Gamma_{g}^{\prime}:{ }^{2 s+1} \Gamma_{u}\right\rangle$ terms (see below).

According to the parity, spin, and orbital quasimomentum selection rules, electric dipole chargetransfer transitions from the ground state ${ }^{4} A_{2 g}$ of $(\mathrm{CrO})_{6}^{9-}$ complex are allowed only into the ${ }^{4} T_{2 u}$ states of $\tilde{\gamma}_{2 p}^{1} t_{2 g}^{4}$ and $\tilde{\gamma}_{2 p}^{1} t_{2 g}^{3} e_{g}$ configurations (transitions into the $t_{2 g}$ and $e_{g}$ shells, respectively). The higher-energy
MOs of the $2 p$ type contain two MOs with the $t_{1 u}$ $\left(t_{1 u}(\pi), t_{1 u}(\sigma)\right)$ symmetry and one MO of the $t_{2 u}(\pi)$ symmetry. Therefore, six low-energy single-electron charge-transfer transitions arise: $t_{2 u}(\pi), t_{1 u}(\pi)$, $t_{1 u}(\sigma) \rightarrow t_{2 g}$ (the calculated energies are 3.1, 3.9, and $5.1 \mathrm{eV}$ [11] for the $\left(\mathrm{FeO}_{6}\right)^{9-}$ complex in orthoferrite) and $t_{2 u}(\pi), t_{1 u}(\pi), t_{1 u}(\sigma) \rightarrow e_{g}(4.4,5.3$, and $6.4 \mathrm{eV}$ [11]).

These single-electron transitions induce the following electric dipole many-electron charge-transfer transitions of the ${ }^{4} A_{2 g} \rightarrow{ }^{4} T_{2 u}$ type:

$$
\gamma_{2 p}^{6}{ }^{1} A_{1 g} ; t_{2 g}^{3}{ }^{4} A_{2 g}:{ }^{4} A_{2 g} \rightarrow \gamma_{2 p}^{5}{ }^{2} \mathbf{r}_{u} ; t_{2 g}^{4} T_{1 g}:{ }^{4} T_{2 u}
$$

(single-electron charge-transfer $\gamma_{2 p} \rightarrow t_{2 g}$ transition) and

$$
\begin{gathered}
\gamma_{2 p}^{6}{ }^{1} A_{1 g} ; t_{2 g}^{3}{ }^{4} A_{2 g}:{ }^{4} A_{2 g} \rightarrow \gamma_{2 p}^{5}{ }^{2} \boldsymbol{\Gamma}_{u} ; \\
t_{2 g}^{3}{ }^{4} A_{2 g} ; e_{g}^{12} E_{g}\left({ }^{2 \sigma+1} E_{g}\right):{ }^{4} T_{2 u}
\end{gathered}
$$

(single-electron charge-transfer $\gamma_{2 p} \rightarrow e_{g}$ transition). Here, $\sigma$ takes values of 1 and 2 .

The measure of transition intensity is known to be the line strength: the squared modulus of reduced matrix element (RME) of the operator responsible for the transition between states. For the operator of electric dipole moment we calculated the RMEs on manyelectron wave functions of the final and initial configurations for the $\gamma_{2 p} \rightarrow t_{2 g}$ charge transfer:

$$
\begin{gathered}
\left\langle\gamma_{2 p}^{5}{ }^{2} \boldsymbol{\Gamma}_{u} ; t_{2 g}^{4}{ }^{3} T_{1 g}:{ }^{4} T_{2 u}\|\mathbf{d}\| \gamma_{2 p}^{6}{ }^{1} A_{1 g} ; t_{2 g}^{3}{ }^{4} A_{2 g}:{ }^{4} A_{2 g}\right\rangle \\
=3(-1)^{j(\mathbf{r})}\left\{\begin{array}{lll}
T_{1} & T_{2} & \mathbf{\Gamma} \\
T_{1} & T_{2} & A_{2}
\end{array}\right\}\left\langle t_{2 g}\|\mathbf{d}\| \boldsymbol{\Gamma}_{u}\right\rangle
\end{gathered}
$$

and the RMEs corresponding to the single-electron $\gamma_{2 p} \rightarrow e_{g}$ transition:

$$
\begin{gathered}
\left\langle\gamma_{2 p}^{5}{ }^{2} \boldsymbol{\Gamma}_{u} ; t_{2 g}^{3}{ }^{4} A_{2 g} ; e_{g}^{12} E_{g}\left({ }^{2 \sigma+1} E_{g}\right):{ }^{4} T_{2 u}\|\mathbf{d}\| \gamma_{2 p}^{6}{ }^{1} A_{1 g} ; t_{2 g}^{3}{ }^{4} A_{2 g}:{ }^{4} A_{2 g}\right\rangle \\
=(-1)^{j(\mathbf{r})+\sigma+1} \sqrt{3(2 \sigma+1)}\left\{\begin{array}{ccc}
E & E & A_{2} \\
T_{2} & T_{1} & \mathbf{r}
\end{array}\right\}\left\langle e_{g}\|\mathbf{d}\| \boldsymbol{\Gamma}_{u}\right\rangle .
\end{gathered}
$$

In formulas (1) and (2), $j(\mathbf{r})$ is a phase factor, which is even for representations $\mathbf{\Gamma}=A_{1}, E, T_{2}$ of the cube group and odd for $\mathbf{\Gamma}=A_{2}, T_{1}$; the expression in braces is the $6 \mathbf{r}$ symbol.

According to (1), three single-electron chargetransfer $\gamma_{2 p} \rightarrow t_{2 g}$ transitions induce three many-electron transitions of the ${ }^{4} A_{2 g} \rightarrow{ }^{4} T_{2 u}$ symmetry. The RMEs of electric dipole moment on many-electron wave functions are $\left(-\left\langle t_{2 g}\|\mathbf{d}\| \mathbf{r}_{u}\right\rangle\right)$ for $\mathbf{r}=t_{1}$ and $\mathbf{r}=t_{2}$.
Three single-electron charge-transfer $\gamma_{2 p} \rightarrow e_{g}$ transitions are responsible for six many-electron transitions of the ${ }^{4} A_{2 g} \rightarrow{ }^{4} T_{2 u}$ type; the RMEs of these transitions, calculated according to (2), are listed in the table.

The results obtained can be parameterized [7] with neglect of multicenter integrals in calculation of RMEs; then all RMEs can be expressed in terms of effective covalence parameters $\lambda_{\sigma}$ and $\lambda_{\pi}$ for $\sigma$ and $\pi$ bonds, respectively. In this RME approximation, 
$\left\langle t_{2 u}(\pi)\|\mathbf{d}\| e_{g}\right\rangle,\left\langle t_{1 u}(\pi)\|\mathbf{d}\| e_{g}\right\rangle$, and $\left\langle t_{1 u}(\sigma)\|\mathbf{d}\| t_{2 g}\right\rangle$ become zero. In terms of [7], the corresponding charge-transfer $t_{2 u}(\pi) \rightarrow e_{g}, t_{1 u}(\pi) \rightarrow e_{g}, t_{1 u}(\sigma) \rightarrow t_{2 g}$, transitions are denoted as "weak," in contrast to the "strong" $t_{1 u}(\sigma) \rightarrow e_{g}, t_{2 u}(\pi), t_{1 u}(\pi) \rightarrow t_{2 g}$ transitions. If this approximation leads to a nonzero RME value, it is valid up to the first order of smallness with respect to the cation-anion overlap integrals.

\section{SIMULATION OF THE CHARGE-TRANSFER TRANSITION BAND IN CHROMITES}

The above theoretical concepts will be used to simulate the optical spectra of perovskite-type chromites $R \mathrm{CrO}_{3}$.

Depending on the energy-gap nature, insulating $3 d$ systems are divided into two groups: Mott-Hubbard and charge-transfer insulators [12]. In the former, the energy gap is formed due to the transitions between the lower and upper Hubbard bands $(U<\Delta)$, whereas in second-group insulators the energy gap is due to the charge-transfer transitions between the upper filled ligand band and the upper Hubbard band $(U>\Delta)$. Actually, in both cases one can speak about chargetransfer transitions, corresponding to two- and onecenter excitons, respectively.

Generally, the energy gap for the $3 d$ transition metals in the end $(\mathrm{Cu}, \mathrm{Ni}$, etc.) and in the beginning ( $\mathrm{Ti}$, $\mathrm{V}$, etc.) of the series is related to the charge-transfer transitions of the $p \rightarrow d$ type $(U>\Delta)$ and the $d \rightarrow d$ type, respectively. Some real situations deviate from this scheme. For example, $\mathrm{CuO}$ and $\mathrm{La}_{2} \mathrm{CuO}_{4}$ cuprates are traditionally considered to be Mott-Hubbard insulators; however, the electron-energy loss spectroscopy data indicate dual nature of their energy gap, which is related to both two- and one-center chargetransfer excitons [13].

Our approach to chromites will be based on the concept of charge-transfer insulators. Let us introduce the following model assumptions.

(i) Since a charge-transfer transition results in the formation of $3 d^{4}$ configuration of the central $\mathrm{Cr}$ ion in $\left(\mathrm{CrO}_{6}\right)^{9-}$ complex, which formally corresponds to the $\mathrm{Mn}^{3+}$ ion, the Tanabe-Sugano diagram of the excited charge-transfer configuration $3 d^{4}$ (see below) is calculated using the Racah parameters $B$ and $C$ for the $\mathrm{Mn}^{3+}$ ion in the ground state [14] in octahedral oxygen environment.

(ii) The energies of nonbonding oxygen states in the $\left(\mathrm{CrO}_{6}\right)^{9-}$ complex are assumed to be equal to those in the $\left(\mathrm{FeO}_{6}\right)^{9-}$ complex [11]. This assumption is well grounded, because the energies of oxygen states depend mainly on the crystalline environment.
Reduced matrix elements of the electric dipole moment operator for many-electron charge-transfer transitions, induced by single-electron $t_{2 u}, t_{1 u} \longrightarrow 3 d e_{g}$ transitions

\begin{tabular}{c|c|c}
\hline$\gamma$ & $\sigma$ & $\mathrm{RME}$ \\
\hline$T_{2}$ & 2 & $\sqrt{5 / 2}\left\langle e_{g}\|\mathbf{d}\| t_{2 u}\right\rangle$ \\
$T_{2}$ & 1 & $-\sqrt{3 / 2}\left\langle e_{g}\|\mathbf{d}\| t_{2 u}\right\rangle$ \\
$T_{1}$ & 2 & $-\sqrt{5 / 2}\left\langle e_{g}\|\mathbf{d}\| t_{1 u}\right\rangle$ \\
$T_{1}$ & 1 & $\sqrt{3 / 2}\left\langle e_{g}\|\mathbf{d}\| t_{1 u}\right\rangle$ \\
\hline
\end{tabular}

Thus, the energy spectrum of single-electron charge-transfer transitions depends on the energies of cation orbitals $3 d t_{2 g}, e_{g}$; within our model, it should shift rigidly with a change in these energies. This conclusion also holds true for many-electron chargetransfer transitions. The calculated theoretical spectrum was referenced to the experimental one using the fundamental optical absorption edge; thus, the position of all peaks in the calculated spectrum was unambiguously determined.

To determine the energies of many-electron charge-transfer transitions and the transition intensities, with allowance for the mixing of charge-transfer states, we obtained the Tanabe-Sugano diagram for the $3 d^{4}$ configuration with the parameters $B=0.12 \mathrm{eV}$ and $\gamma=C / B=4.61$ [14] (Fig. 1, central panel).

The degeneracy of the $d^{4}$ level of a free ion is removed by electrostatic repulsion of electrons (Fig. 1, left panel) and the crystal field of ligands (Fig. 1, central panel). The pattern of level splitting in the presence of crystal field with a decrease in electronic correlations (i.e., at Racah parameters $B, C \rightarrow 0$ ) is shown in Fig. 1 (the right panel).

Analysis of the spectra in single-electron band models in terms of $2 p \rightarrow 3 d t_{2 g}$ and $2 p \rightarrow 3 d e_{g}$ transitions is equivalent to the consideration of simplified pattern (right panel) instead of the complete one (the central panel).

At the crystal-field parameter $D q=0.25 \mathrm{eV}$ [14], we obtain a strong band of charge-transfer transitions, approximately $8 \mathrm{eV}$ wide (from $\approx 2$ to $\approx 10 \mathrm{eV}$ ). Each single-electron $t_{2 u}(\pi), t_{1 u}(\pi), t_{1 u}(\sigma) \rightarrow t_{2 g}$ transition induces seven many-electron charge-transfer transitions, and each single-electron $t_{2 u}(\pi), t_{1 u}(\pi)$, $t_{1 u}(\sigma) \rightarrow e_{g}$ transition induces four many-electron charge-transfer transitions. In total, the band consists of 33 lines.

We took into account the corrections to the level energies due to the Coulomb quadrupole-quadrupole interaction of the $2 p$ hole with the $3 d$ shell (Fig. 2). These correction turned out to be insignificant ( $\approx 0.1 \mathrm{eV}$; see Appendix). 


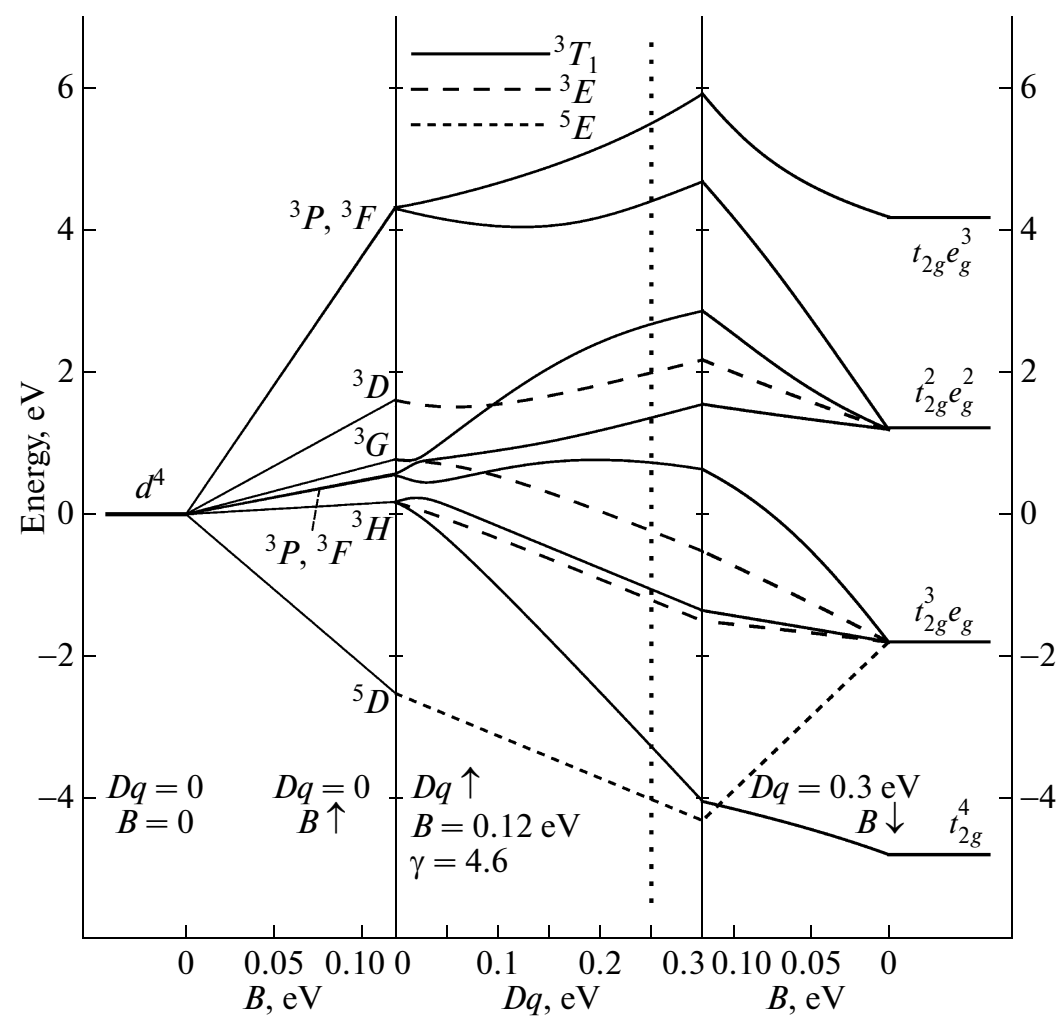

Fig. 1. Correlation diagram for the excited charge-transfer $3 d^{4}$ configuration in an octahedral crystal field. The degeneracy of the $d^{4}$ level of the free ion is removed by the electron-electron Coulomb interaction (left panel) and crystal field (central panel). The central panel shows the Tanabe-Sugano diagram with the Racah parameters $B=0.12 \mathrm{eV}$ and $\gamma=C / B=4.61$ (only the terms significant for charge-transfer transitions are shown). The vertical dotted line corresponds to the value $D q=0.25 \mathrm{eV}$ [14], which was used to calculate the model spectrum (see Fig. 3). Energy-level splitting in crystal field with a decrease in the Racah parameters $B$ and $C$ is shown in the right panel.

We simulated the band of electric dipole chargetransfer transitions in the optical spectrum of orthochromite (within the model assumptions, the characteristic features of a specific compound manifest themselves in only the value of the rigid shift of all spectral lines). As was noted above, the contribution of the charge-transfer $t_{2 u}(\pi) \rightarrow e_{g}, t_{1 u}(\pi) \rightarrow e_{g}$, and $t_{1 u}(\sigma) \rightarrow t_{2 g}$ transitions becomes zero as a result of parameterization; therefore, to take them into account, we will artificially introduce the corresponding covalence parameters $\lambda_{\pi \sigma}=\lambda_{\sigma \pi}$. Reasonable physical considerations (different values of overlap integrals due to the different bond geometry and separation of transitions into strong and weak) justify the relation between the covalence parameters that we used to model spectra: $\lambda_{\pi}^{2}=1 / 5 \lambda_{\sigma}^{2}$ and $\lambda_{\pi \sigma}^{2}=1 / 10 \lambda_{\sigma}^{2}$.

Line half-width $\Gamma$ was assumed to be $0.4 \mathrm{eV}$ for many-electron charge-transfer transitions corresponding to weak single-electron $t_{2 u}(\pi) \rightarrow e_{g}$, $t_{1 u}(\pi) \rightarrow e_{g}$, and $t_{1 u}(\sigma) \rightarrow t_{2 g}$ transitions; $0.8 \mathrm{eV}$ in the case of strong $t_{2 u}(\pi), t_{1 u}(\pi) \rightarrow t_{2 g}$ transitions; and $1.2 \mathrm{eV}$ for the strong $t_{1 u}(\sigma) \rightarrow e_{g}$ transition. Note that the model results depend only slightly on the choice of $\Gamma$. The relationship between the chosen $\Gamma$ values is justified by the fact that specifically strong electronic transitions have a high oscillator strength and specifically the latter is proportional to the line half-width [15].

The spectrum was simulated using the Lorentzian profile

$$
I(\omega)=4 \omega \omega_{0} \Gamma /\left[\left(\omega^{2}-\omega_{0}^{2}-\Gamma^{2}\right)^{2}+4 \omega^{2} \Gamma^{2}\right],
$$

where $\omega_{0}$ is the transition frequency.

The calculation results are shown in Fig. 3. The main panel presents the calculated spectral dependence of the total contribution of all (both strong and weak) electric dipole charge-transfer transitions to imaginary part $\varepsilon_{2}$ of the diagonal component of permittivity tensor $\varepsilon=\varepsilon_{1}+i \varepsilon_{2}$. For comparison, the inset shows the experimental spectra $\varepsilon_{2}$ for $\mathrm{Lu}_{0.85} \mathrm{Y}_{0.15} \mathrm{CrO}_{3}$ [16], $\mathrm{YCrO}_{3}$ [17], and $\mathrm{LaCrO}_{3}$ [17] (curves 1, 2, and 3 , respectively). The calculated spectrum is in reasonable agreement with the experimental one. 


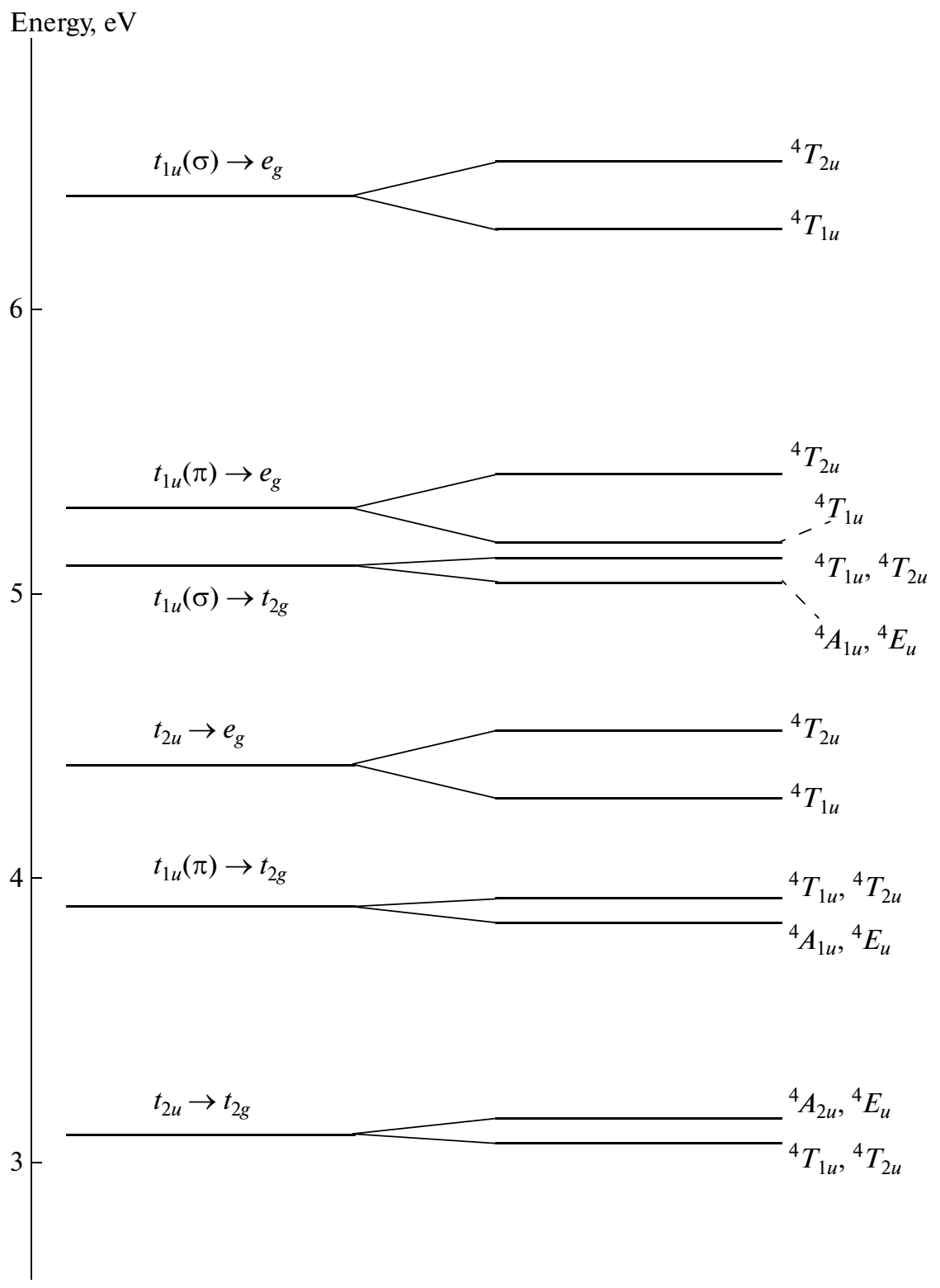

Fig. 2. Fragment of the energy structure of charge-transfer configurations in the $\left(\mathrm{CrO}_{6}\right)^{9-}$ complex, with allowance for the Coulomb (quadrupole-quadrupole) interaction (level splitting is somewhat exaggerated for clearness). The ${ }^{4} T_{2 u}$ states are final for the allowed electric dipole charge-transfer ${ }^{4} A_{2 g} \rightarrow{ }^{4} T_{2 u}$ transitions. The final charge-transfer states induced by the $t_{2 u}(\pi)$, $t_{1 u}(\pi) \rightarrow t_{2 g}$ transitions are almost degenerate pairwise; the states arising at the charge-transfer $t_{1 u}(\sigma) \rightarrow t_{2 g}$ transition are characterized by exact degeneracy (see Appendix, Subsection 1). The energy values are related to the $\left(\mathrm{Fe} \mathrm{O}_{6}\right)^{9-}$ complex [11].

We investigated the dependence of the $\varepsilon_{2}$ spectrum on the crystal-field parameter $D q$ (Fig. 4). The change in $D q$ not only affects the relative heights of peaks in the spectrum, but also leads to its complete transformation.

\section{CONCLUSIONS}

We considered the specific features of states and charge-transfer $\mathrm{O} 2 p \rightarrow \mathrm{Cr} 3 d$ transitions in the octa- hedral complex $\left(\mathrm{CrO}_{6}\right)^{9-}$ within the cluster approximation, which combines the concepts of crystal-field theory and the MO method. One alternative to these (intracenter) transitions is intercenter charge-transfer transitions involving neighboring complexes:

$$
\left(\mathrm{CrO}_{6}\right)^{9-}+\left(\mathrm{CrO}_{6}\right)^{9-} \rightarrow\left(\mathrm{CrO}_{6}\right)^{10-}+\left(\mathrm{CrO}_{6}\right)^{8-} .
$$

These transitions have not been considered here. 


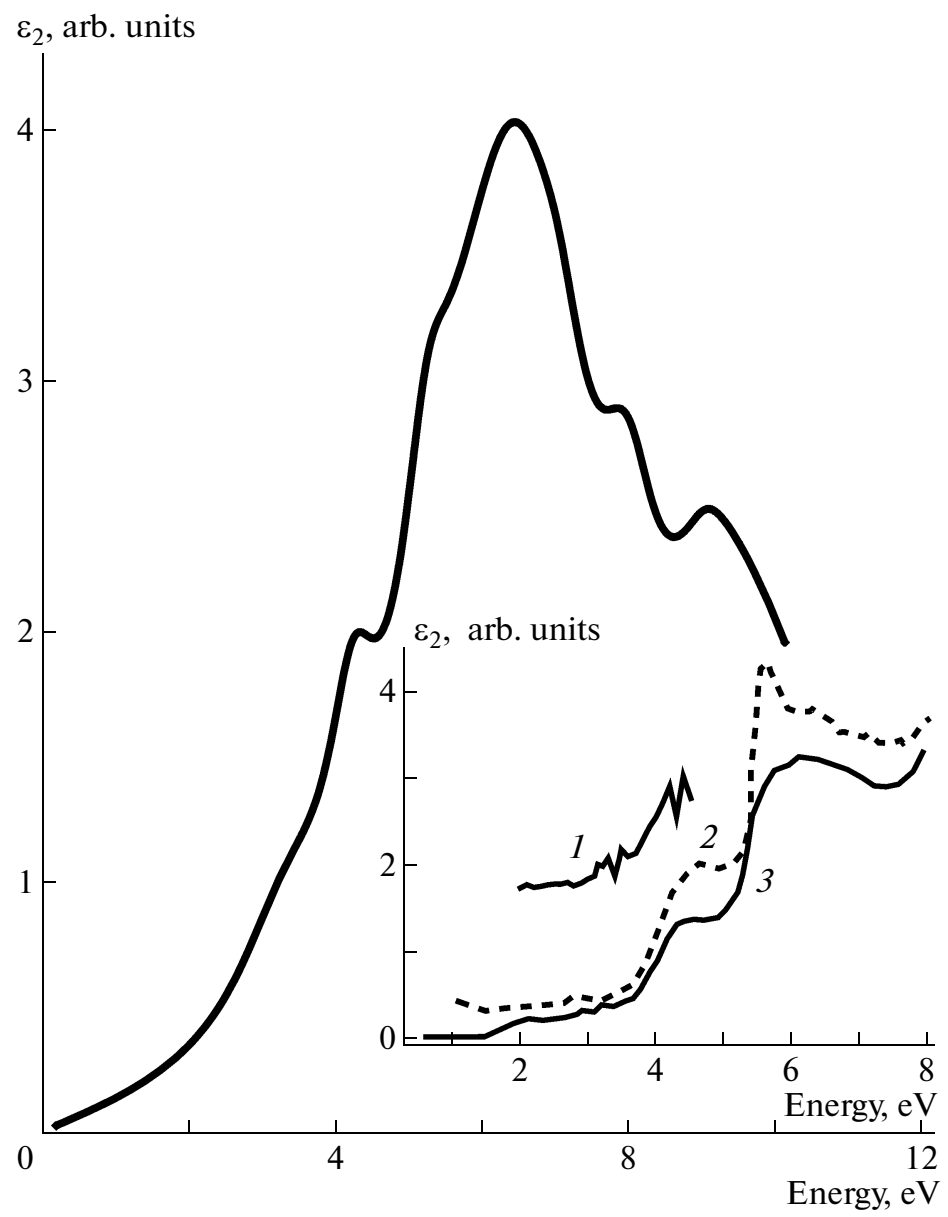

Fig. 3. Result of simulation of the spectral dependence of the band of charge-transfer $\mathrm{O} 2 p \rightarrow \mathrm{Cr} 3 d$ transitions in chromite. The main panel shows the total contribution of all allowed electric dipole charge-transfer transitions to the imaginary part $\varepsilon_{2}$ of the diagonal component of the permittivity tensor $\varepsilon=\varepsilon_{1}+i \varepsilon_{2}$. For comparison, the inset shows the experimental $\varepsilon_{2}$ spectra for chromites $\mathrm{Lu}_{0.85} \mathrm{Y}_{0.15} \mathrm{CrO}_{3}$ [16], $\mathrm{YCrO}_{3}$ [17], and $\mathrm{LaCrO}_{3}$ [17] (lines 1, 2, and 3, respectively).

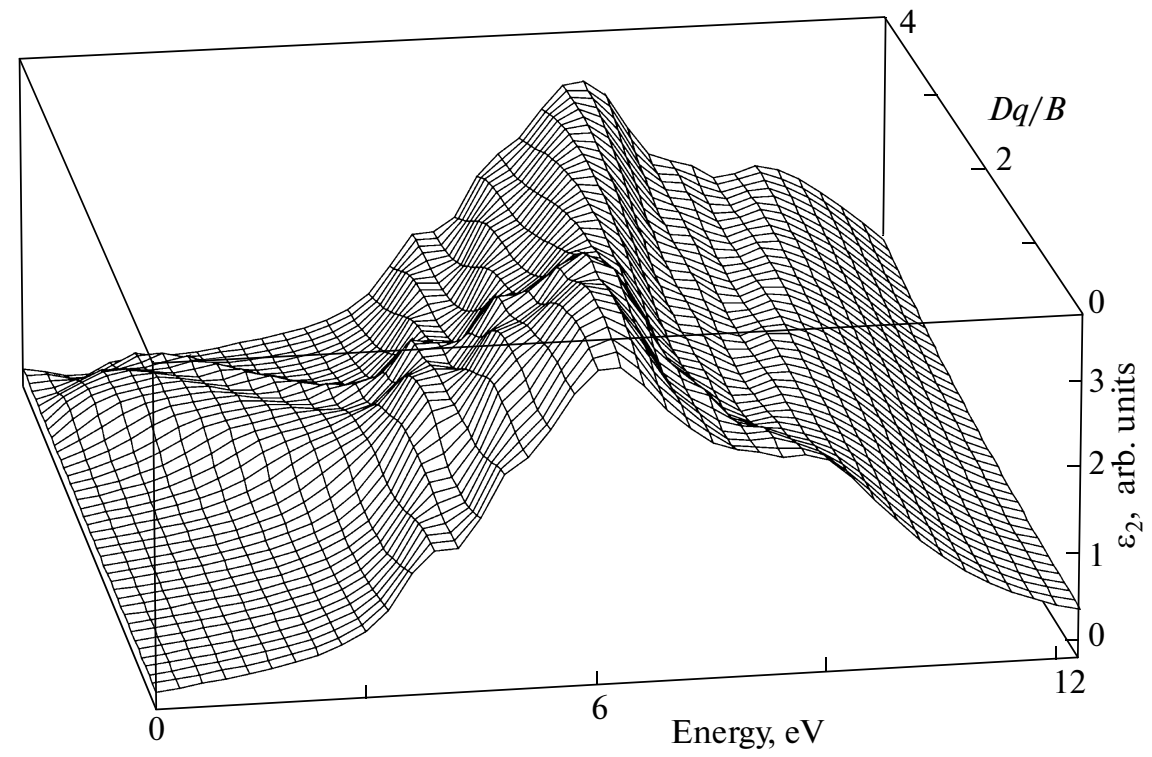

Fig. 4. Result of simulation of the spectral dependence of the band of charge-transfer $\mathrm{O} 2 p \rightarrow \mathrm{Cr} 3 d$ transitions in chromite. The total contribution of all allowed electric dipole charge-transfer transitions to the imaginary part $\varepsilon_{2}$ of the diagonal component of the permittivity tensor is shown as a function of crystal field strength. 
The Racah algebra for the rotation and cube groups was used to calculate the RME operator of electric dipole moment on many-electron wave functions, which are the initial and final states for charge-transfer transitions. Parameterization of the results allowed us to calculate the relative intensities of many-electron charge-transfer transitions. Their energies were obtained within the crystal-field theory.

The corrections to level energies due to the Coulomb quadrupole interaction between the $2 p$ hole and $3 d$ shell were calculated. The influence of this interaction on the optical spectrum turned out to be insignificant.

Simulation of the chromite optical spectrum revealed the presence of a complex band of chargetransfer transitions, composed of 33 lines, with a main peak of around $7 \mathrm{eV}$ and additional peaks in the ranges of $4-5$ and $8-9 \mathrm{eV}$. The total band width is $\approx 8 \mathrm{eV}$. The influence of the crystal field and electronic correlations on the spectrum structure was investigated.

Despite the roughness of the model assumptions, the calculated spectrum is in qualitative agreement with the experimental one. It would be rather difficult to explain the optical spectra of chromites (and other $3 d$ oxides [8-10]) beyond the concept of many-electron charge-transfer transitions.

\section{COULOMB SPLITTING \\ OF CHARGE-TRANSFER STATES}

The Coulomb interaction between the $2 p$ electrons of $\mathrm{O}^{2-}$ ligand and $3 d$ electrons of the central $\mathrm{Cr}^{3+}$ ion in the $\left(\mathrm{CrO}_{6}\right)^{9-}$ cluster is written conventionally:

$$
V_{C}=\sum_{i, j} e^{2} / r_{i j}
$$

where $\mathbf{r}_{i j}=\mathbf{R}_{12}+\mathbf{r}_{i}-\mathbf{r}_{j}$ and the radii vectors of electrons $\left(\mathbf{r}_{i}\right.$ and $\mathbf{r}_{j}$ ) of the first and second ions are counted from their centers.

Using multipole expansion, we transform this formula as follows:

$$
\begin{aligned}
V_{C} & =e^{2} \sum_{i, j} \sum_{\substack{k_{1}, q_{1} \\
k_{2}, q_{2}}} \delta_{k, k_{1}+k_{2}} \frac{r_{i}^{k_{1}} r_{j}^{k_{2}}}{R_{12}^{k+1}}(-1)^{k_{1}}\left(\begin{array}{lll}
k_{1} & k_{2} & k \\
q_{1} & q_{2} & q
\end{array}\right) \\
& \times \sqrt{\frac{(2 k+1) !}{\left(2 k_{1}\right) !\left(2 k_{2}\right) !}} C_{q_{1}}^{k_{1}}\left(\mathbf{r}_{i}\right) C_{q_{2}}^{k_{2}}\left(\mathbf{r}_{j}\right) C_{q}^{k}\left(\mathbf{R}_{12}\right),
\end{aligned}
$$

where $C_{q}^{k}(\mathbf{r})=\sqrt{4 \pi /(2 k+1)} Y_{k q}(\mathbf{r})$ is a spherical tensor and the expression in parentheses is the $3 \mathrm{jm}$ symbol.

In the first order of the perturbation theory, the Coulomb splitting of charge-transfer states is determined by the diagonal matrix element $V_{C}$ on the corresponding wave functions:

$$
\Delta E(\Gamma)=\left\langle\gamma_{2 p}^{5}{ }^{2} \gamma_{u} ; d^{4}\left(t_{2 g}^{4}{ }^{3} T_{1 g} \text { or } t_{2 g}^{3} e_{g}^{13 ; 5} E_{g}\right):{ }^{2 s+1} \Gamma\left|V_{C}\right| \gamma_{2 p}^{5}{ }^{2} \gamma_{u} ; d^{4}\left(t_{2 g}^{4}{ }^{3} T_{1 g} \text { or } t_{2 g}^{3} e_{g}^{13 ; 5} E_{g}\right):{ }^{2 s+1} \Gamma\right\rangle
$$

where $\Gamma=\gamma \times T_{1}($ or $\gamma \times E)$. Substituting formula (A.1) into (A.2) and performing transformations, we obtain the RMEs $\left\langle\ell_{1}\left\|C^{k_{1}}\right\| \ell_{1}\right\rangle$ and $\left\langle\ell_{2}\left\|C^{k_{2}}\right\| \ell_{2}\right\rangle$ of the spherical tensors on the orbital states of $\mathrm{O}^{2-}$ and $\mathrm{Cr}^{3+}$ ions. Since $\left\langle\ell\left\|C^{\ell^{\prime}}\right\| \ell^{\prime \prime}\right\rangle \neq 0$ only when the sum $\ell+\ell^{\prime}+\ell^{\prime \prime}$ is even, consideration of the dipole-dipole interaction $\left(k_{1}=k_{2}=1\right)$ does not lead to splitting of charge-transfer states $(\Delta E=0)$. The nonzero $\Delta E$ value arises in the quadrupole-quadrupole approximation $\left(k_{1}=k_{2}=2\right)$. The higher orders of multipole expansion do not contribute to $\Delta E$, because the RMEs $\left\langle\ell\left\|C^{\ell^{\prime}}\right\| \ell^{\prime \prime}\right\rangle$ become zero according to the triangle rule. Substituting $k_{1}=k_{2}$ $=2$ and $k=4$ into (A1) and passing to cubic tensors, we obtain the following expression for the Coulomb (quadrupole-quadrupole) interaction:

$$
\begin{gathered}
V_{C}=\sqrt{70} e^{2} \frac{r_{2 p}^{2} r_{3 d}^{2}}{R_{12}^{5}} \sum_{\gamma=E, T_{2}}[\gamma]^{-1 / 2} \\
\times N_{\gamma \gamma A_{1}}^{224}\left(C^{2 \gamma}\left(\mathbf{r}_{1}\right) C^{2 \gamma}\left(\mathbf{r}_{2}\right)\right) C_{0}^{4 A_{1}}\left(\mathbf{R}_{12}\right) .
\end{gathered}
$$

In the latter formula,

$$
\Delta=e^{2}\left\langle r^{2}\right\rangle_{2 p}\left\langle r^{2}\right\rangle_{3 d} / R_{12}^{5}
$$

The normalizing factor $N_{\gamma \gamma A_{1}}^{224}$ takes values of $\sqrt{3 / 5}$ for $\gamma=E$ and $(-\sqrt{2 / 5})$ for $\gamma=T_{2}$.

Using formulas (A.2) and (A.3) and performing necessary transformations, we obtain the correction to the energy of the ${ }^{2 s+1} \Gamma$ term due to the Coulomb quadrupole-quadrupole interaction in the form

$$
\Delta E(\Gamma)=\sum_{\gamma=E, T_{2}}(-1)^{\Gamma}\left\{\begin{array}{ccc}
\gamma_{u} & G & \Gamma \\
G & \gamma_{u} & \gamma
\end{array}\right\} \Delta_{\gamma},
$$

where the term $G$ is related to the $3 d$ subsystem $\left({ }^{3} T_{1 g}\right.$ or $\left.{ }^{3 ; 5} E_{g}\right)$,

$$
\begin{aligned}
& \Delta_{\gamma}=\sqrt{70} \Delta[\gamma]^{-1 / 2} N_{\gamma \gamma A_{1}}^{224} C_{0}^{4 A_{1}}(\mathbf{R}) \\
& \quad \times\left\langle\gamma_{2 p}^{5}\left\|C^{2 \gamma}\right\| \gamma_{2 p}^{5}\right\rangle\left\langle G\left\|C^{2 \gamma}\right\| G\right\rangle .
\end{aligned}
$$


Thus, to calculate energy corrections $\Delta E(\Gamma)$, one needs RMEs of cubic tensor operator $C^{2 \gamma}$ on the wave functions $\gamma_{2 p}^{5}$ and $G$ (see Appendix, Subsection 2).

According to formula (A.4), we obtain the following values.

For the $t_{2 u} \rightarrow t_{2 g}$ transition:

$$
\begin{gathered}
\Delta E\left(A_{2 u}\right)=\frac{17 \sqrt{3 \cdot 7 \cdot 11 \cdot 13}}{2^{3} \cdot 3^{2} \cdot 5 \cdot 13} \Delta=0.199 \Delta, \\
\Delta E\left(E_{u}\right)=\frac{31 \sqrt{3 \cdot 7 \cdot 11 \cdot 13}}{2^{4} \cdot 3^{2} \cdot 5 \cdot 13} \Delta=0.182 \Delta, \\
\Delta E\left(T_{1 u}\right)=-\frac{\sqrt{3 \cdot 7 \cdot 11 \cdot 13}}{2^{4} \cdot 3 \cdot 13} \Delta=-0.088 \Delta, \\
\Delta E\left(T_{2 u}\right)=-\frac{17 \sqrt{3 \cdot 7 \cdot 11 \cdot 13}}{2^{4} \cdot 3^{2} \cdot 5 \cdot 13} \Delta=-0.100 \Delta .
\end{gathered}
$$

Note the approximate degeneracy of the $A_{2 u}$ and $E_{u}$ and $T_{1 u}$ and $T_{2 u}$ pairs.

For the $t_{1 u}(\pi) \rightarrow t_{2 g}$ transition,

$$
\begin{gathered}
\Delta E\left(A_{1 u}\right)=-\frac{17 \sqrt{3 \cdot 7 \cdot 11 \cdot 13}}{2^{3} \cdot 3^{2} \cdot 5 \cdot 13} \Delta=-0.199 \Delta, \\
\Delta E\left(E_{u}\right)=-\frac{31 \sqrt{3 \cdot 7 \cdot 11 \cdot 13}}{2^{4} \cdot 3^{2} \cdot 5 \cdot 13} \Delta=-0.182 \Delta, \\
\Delta E\left(T_{1 u}\right)=\frac{17 \sqrt{3 \cdot 7 \cdot 11 \cdot 13}}{2^{4} \cdot 3^{2} \cdot 5 \cdot 13} \Delta=0.100 \Delta, \\
\Delta E\left(T_{2 u}\right)=-\frac{\sqrt{3 \cdot 7 \cdot 11 \cdot 13}}{2^{4} \cdot 3 \cdot 13} \Delta=0.088 \Delta .
\end{gathered}
$$

Note the approximate degeneracy of the $A_{1 u}$ and $E_{u}$ and $T_{1 u}$ and $T_{2 u}$ pairs.

For the $t_{2 u} \rightarrow e_{g}$ transition,

$$
\begin{gathered}
\Delta E\left(T_{1 u}\right)=-0.4 \Delta, \\
\Delta E\left(T_{2 u}\right)=0.4 \Delta .
\end{gathered}
$$

For the $t_{1 u}(\sigma) \rightarrow t_{2 g}$ transition,

$$
\begin{gathered}
\Delta E\left(A_{1 u}\right)=-\frac{2 \sqrt{3 \cdot 7 \cdot 11 \cdot 13}}{3^{2} \cdot 5 \cdot 13} \Delta=-0.187 \Delta, \\
\Delta E\left(E_{u}\right)=-\frac{2 \sqrt{3 \cdot 7 \cdot 11 \cdot 13}}{3^{2} \cdot 5 \cdot 13} \Delta=-0.187 \Delta, \\
\Delta E\left(T_{1 u}\right)=\frac{\sqrt{3 \cdot 7 \cdot 11 \cdot 13}}{3^{2} \cdot 5 \cdot 13} \Delta=0.094 \Delta \\
\Delta E\left(T_{2 u}\right)=\frac{\sqrt{3 \cdot 7 \cdot 11 \cdot 13}}{3^{2} \cdot 5 \cdot 13} \Delta=0.094 \Delta .
\end{gathered}
$$

Note the exact degeneracy of the $A_{1 u}$ and $E_{u}$ and $T_{1 u}$ and $T_{2 u}$ pairs.

For the $t_{1 u}(\pi) \rightarrow e_{g}$ transition,

$$
\begin{gathered}
\Delta E\left(T_{1 u}\right)=-0.4 \Delta, \\
\Delta E\left(T_{2 u}\right)=0.4 \Delta .
\end{gathered}
$$

For the $t_{1 u}(\sigma) \rightarrow e_{g}$ transition,

$$
\begin{gathered}
\Delta E\left(T_{1 u}\right)=-0.4 \Delta, \\
\Delta E\left(T_{2 u}\right)=0.4 \Delta .
\end{gathered}
$$

Estimates of $\Delta$ and $\Delta E(\Gamma)$ show that the energy corrections can barely exceed $0.1 \mathrm{eV}$. Thus, the Coulomb interaction between the $2 p$ electrons of $\mathrm{O}^{2-}$ ligands and the $3 d$ electrons of the central $\mathrm{Cr}^{3+}$ ion in the $\left(\mathrm{CrO}_{6}\right)^{9-}$ complex cannot significantly affect the optical spectra of chromites.

\section{CALCULATION OF REDUCED MATRIX ELEMENTS}

\section{Reduced matrix elements on $3 d$ functions}

The ground state of the $d^{4}$ configuration is ${ }^{3} H$. There are two versions of relation between the harmonics $|k q\rangle$ in the rotation group and $|\gamma v\rangle$ in the cube group at $k=5$.

The first version:

$$
\begin{gathered}
\left|T_{1} 1\right\rangle=\sqrt{\frac{5}{128}}|55\rangle+\sqrt{\frac{21}{64}}|51\rangle-\sqrt{\frac{81}{128}}|5-3\rangle, \\
\left|T_{1} 0\right\rangle=\frac{1}{\sqrt{2}}|54\rangle+\frac{1}{\sqrt{2}}|5-4\rangle, \\
\left|T_{1}-1\right\rangle=-\sqrt{\frac{81}{128}}|53\rangle+\sqrt{\frac{21}{64}}|5-1\rangle+\sqrt{\frac{5}{128}}|5-5\rangle .
\end{gathered}
$$

The second version (which is lower in energy; specifically this version is used below):

$$
\begin{gathered}
\left|T_{1} 1\right\rangle=\sqrt{\frac{63}{128}}|55\rangle+\sqrt{\frac{15}{64}}|51\rangle+\sqrt{\frac{35}{128}}|5-3\rangle, \\
\left|T_{1} 0\right\rangle=|50\rangle, \\
\left|T_{1}-1\right\rangle=\sqrt{\frac{35}{128}}|53\rangle+\sqrt{\frac{15}{64}}|5-1\rangle+\sqrt{\frac{63}{128}}|5-5\rangle .
\end{gathered}
$$

For the $t_{2 u} \rightarrow t_{2 g}$ transition,

$$
\begin{gathered}
\left\langle t_{2 g}^{4}{ }^{3} T_{1 g}\left\|C^{2 E}\right\| t_{2 g}^{4}{ }^{3} T_{1 g}\right\rangle=-\frac{2}{3} \sqrt{\frac{11}{7 \cdot 13}}, \\
\left\langle t_{2 g}^{4}{ }^{3} T_{1 g}\left\|C^{2 T_{2}}\right\| t_{2 g}^{4}{ }^{3} T_{1 g}\right\rangle=-\frac{\sqrt{6 \cdot 7 \cdot 11 \cdot 13}}{2^{3} \cdot 3 \cdot 7 \cdot 13} .
\end{gathered}
$$

For the $\gamma_{2 p} \rightarrow e_{g}$ transition,

$\left\langle t_{2 g}^{3}{ }^{4} A_{2 g} ; e_{g}^{12} E_{g}:{ }^{3 ; 5} E_{g}\left\|C^{2 E}\right\| t_{2 g}^{3}{ }^{4} A_{2 g} ; e_{g}^{12} E_{g}:{ }^{3 ; 5} E_{g}\right\rangle=4 / 7$, $\left\langle t_{2 g}^{34} A_{2 g} ; e_{g}^{12} E_{g}:{ }^{3 ; 5} E_{g}\left\|C^{2 T_{2}}\right\| t_{2 g}^{3}{ }^{4} A_{2 g} ; e_{g}^{12} E_{g}:{ }^{3 ; 5} E_{g}\right\rangle=0$.

The last result is obvious without calculations, because in this case the generalized triangle rule is violated: $T_{2} \notin E \times E$. 
Reduced matrix elements on ligand wave functions

$\left\langle t_{2 u}^{5}\left\|C^{2 E}\right\| t_{2 u}^{5}\right\rangle=-2 \sqrt{3} / 5, \quad\left\langle t_{2 u}^{5}\left\|C^{2 T_{2}}\right\| t_{2 u}^{5}\right\rangle=-3 \sqrt{2} / 10 ;$

$$
\left\langle t_{1 u}^{5}(\pi)\left\|C^{2 E}\right\| t_{1 u}^{5}(\pi)\right\rangle=2 \sqrt{3} / 5,
$$$$
\left\langle t_{1 u}^{5}(\pi)\left\|C^{2 T_{2}}\right\| t_{1 u}^{5}(\pi)\right\rangle=-3 \sqrt{2} / 10 ;
$$

$\left\langle t_{1 u}^{5}(\sigma)\left\|C^{2 E}\right\| t_{1 u}^{5}(\sigma)\right\rangle=2 \sqrt{3} / 5, \quad\left\langle t_{1 u}^{5}(\sigma)\left\|C^{2 T_{2}}\right\| t_{1 u}^{5}(\sigma)\right\rangle=0$.

Let us demonstrate the calculation of RMEs by the example of $\left\langle t_{2 u}^{5}\left\|C^{2 T_{2}}\right\| t_{2 u}^{5}\right\rangle$. First, $\left\langle t_{2 u}^{5}\left\|C^{2 T_{2}}\right\| t_{2 u}^{5}\right\rangle=$ $-\left\langle t_{2 u}\left\|C^{2 T_{2}}\right\| t_{2 u}\right\rangle$ (see, e.g., [18]). According to the Wigner-Eckart theorem for cubic tensors,

$$
\begin{gathered}
\left\langle t_{2 u} 1\left|C_{2}^{2 T_{2}}\right| t_{2 u}-1\right\rangle \\
=(-1)^{j\left(T_{2}\right)-1}\left\langle\begin{array}{ccc}
T_{2} & T_{2} & T_{2} \\
-1 & 2 & -1
\end{array}\right\rangle^{*}\left\langle t_{2 u}\left\|C^{2 T_{2}}\right\| t_{2 u}\right\rangle .
\end{gathered}
$$
bol.

Here, the array in the angle brackets is the $3 \Gamma$ sym-

The cubic-tensor component

$$
C_{2}^{2 T_{2}}=(i / \sqrt{2})\left(-C_{2}^{2}+C_{-2}^{2}\right),
$$

and the wave function $\left|t_{2 u} \pm 1\right\rangle=-\left(\varphi_{1} \pm i \varphi_{2}\right) / \sqrt{2}$, where

$$
\begin{aligned}
& \varphi_{1}=\left(\pi_{1 x}-\pi_{3 x}+\pi_{4 x}-\pi_{6 x}\right) / 2, \\
& \varphi_{2}=\left(\pi_{1 y}-\pi_{2 y}+\pi_{4 y}-\pi_{5 y}\right) / 2
\end{aligned}
$$

(the ligand wave functions are set in the general axes of the $\left(\mathrm{CrO}_{6}\right)^{9-}$ octahedron [14]).

Having neglected the overlap of electronic clouds related to different ligands, one can reduce the matrix element on the left-hand side of (A.6) to a set of integrals of the $\left\langle\pi_{1 x}\left|C_{2}^{2}\right| \pi_{1 x}\right\rangle=\sqrt{6} / 10$ type (its value can easily be calculated from the Wigner-Eckart theorem for the rotation group). Finally, we have $\left\langle t_{2 u} 1\left|C_{2}^{2 T_{2}}\right| t_{2 u}-1\right\rangle=-i \sqrt{3} / 10$. Since $\left\langle\begin{array}{ccc}T_{2} & T_{2} & T_{2} \\ -1 & 2 & -1\end{array}\right\rangle=$ $-i / \sqrt{6}$, (A.6) finally yields $\left\langle t_{2 u}\left\|C^{2 T_{2}}\right\| t_{2 u}\right\rangle=3 \sqrt{2} / 10$.

\section{REFERENCES}

1. A. M. Clogston, J. Phys. 20, 151 (1959).

2. F. J. Kahn, P. S. Pershan, and J. P. Remeika, Phys. Rev. 186, 891 (1969).

3. M. Imada, A. Fujimori, and Y. Tokura, Rev. Mod. Phys. 70, 1039 (1998).

4. K. Shinagawa, Magnetooptics, Ed. by S. Sugano and N. Kojima (Springer, Berlin, 1999), p. 137.

5. A. B. P. Lever, Inorganic Electronic Spectroscopy, 2nd ed. (Elsevier, Amsterdam, 1984; Mir, Moscow, 1987).

6. T. Ido, K. Magoshi, H. Eisaki, and S. Uchida, Phys. Rev. 44, 12094 (1991).

7. A. S. Moskvin, Phys. Rev. B 65, 205113 (2002).

8. A. V. Zenkov, B. B. Krichevtsov, A. S. Moskvin, K. M. Mukimov, R. V. Pisarev, and M. M. Ruvinshtein, Sov. Phys. JETP 69, 792 (1989).

9. Yu. P. Gaidukov, A. V. Zenkov, S. V. Koptsik, G. S. Krinchik, and A. S. Moskvin, JETP Lett. 51, 228 (1990).

10. A. S. Moskvin, A. V. Zenkov, E. A. Ganshina, G. S. Krinchik, and M. M. Nishanova, J. Phys. Chem. Solids 54, 101 (1993).

11. A. I. Likhtenshtein, A. S. Moskvin, and V. A. Gubanov, Sov. Phys. Solid State 24, 2049 (1982).

12. J. Zaanen, G. A. Sawatzky, and J. W. Allen, Phys. Rev. Lett. 55, 418 (1985).

13. A. S. Moskvin, R. Neudert, M. Knupfer, J. Fink, and R. Hayn, Phys. Rev. B 65, 180512(R) (2002).

14. I. B. Bersuker, Electronic Structure and Properties of Coordination Compounds (Khimiya, Leningrad, 1986) [in Russian].

15. J. D. Jackson, Classical Electrodynamics, 3rd ed. (Wiley, New York, 2001), Sect. 16.7.

16. E. A. Gan'shina, Phys. Solid State 35, 174 (1993).

17. T. Arima and Y. Tokura, J. Phys. Soc. Jpn. 64, 2488 (1995).

18. I. I. Sobel'man, Introduction into Theory of Atomic Spectra (GIFML, Moscow, 1963; Oxford University Press, Oxford, 1972). 